\title{
Pilot investigation of the mutation profile of PIK3CA/PTEN genes (PI3K pathway) in grade 3 endometrial cancer
}

\author{
FRANCESCA MALENTACCHI ${ }^{1}$, IRENE TURRINI ${ }^{1}$, FLAVIA SORBI $^{1}$, ELISABETTA PROJETTO ${ }^{2}$, \\ FRANCESCA CASTIGLIONE ${ }^{2}$, MASSIMILIANO FAMBRINI ${ }^{1}$, FELICE PETRAGLIA ${ }^{1}$, \\ SERENA PILLOZZI $^{3 *}$ and IVO NOCI ${ }^{1 *}$ \\ ${ }^{1}$ Department of Biomedical, Experimental and Clinical Sciences, Division of Obstetrics and Gynecology; \\ ${ }^{2}$ Department of Surgery and Translational Medicine, Division of Pathological Anatomy; \\ ${ }^{3}$ Department of Experimental and Clinical Medicine, University of Florence, I-50134 Florence, Italy
}

Received April 16, 2018; Accepted October 9, 2018

DOI: 10.3892/or.2018.6939

\begin{abstract}
Endometrial cancer (EC) comprises a biological and clinical heterogeneous group of tumors. Several genetic alterations are involved in the development and progression of EC, and may be used for targeted therapy, particularly in patients with advanced-stage EC. In the present study, a combined procedure was developed based on polymerase chain reaction (PCR)-high resolution melting analysis (HRMA) and Sanger sequencing for the evaluation of somatic mutations in selected phosphoinositide 3-kinase (PI3K) catalytic subunit $\alpha$ (PIK3CA; exons 1,9 and 21) and phosphatase and tensin homolog (PTEN; exons 5, 6,7 and 8) exons. This combined procedure has the specificity and sensitivity of the two techniques, and overcomes their limitations. A pilot study was performed on 18 selected homogenous EC samples, of grade 3 endometrioid subtype (G3 EEC). First, the feasibility of the combined procedure was investigated to properly identify the presence of somatic mutations on PIK3CA and PTEN, the variations identified were analyzed using Catalogue of Somatic Mutations in Cancer,
\end{abstract}

Correspondence to: Dr Francesca Malentacchi, Department of Biomedical, Experimental and Clinical Sciences, Division of Obstetrics and Gynecology, University of Florence, Viale G.B. Morgagni 50, I-50134 Florence, Italy

E-mail: francesca.malentacchi@gmail.com

*Joint senior authorship

Abbreviations: EC, endometrial cancer; TCGA, The Genome Cancer Atlas; PI3K, phosphoinositide 3-kinase; EEC, endometrioid EC; AKT, protein kinase B; PTEN, phosphatase and tensin homolog; PIK3CA, PI3K catalytic subunit $\alpha$; mTOR, mammalian target of rapamycin; G, grade; SNP, single nucleotide polymorphism; PCR, polymerase chain reaction, HRM, high-resolution melting; HRMA, high-resolution melting analysis; MT, Mutation Taster

Key words: endometrial endometrioid cancer, somatic mutations, phosphoinositide 3-kinase catalytic subunit $\alpha /$ phosphatase and tensin homolog genes, polymerase chain reaction-high-resolution melting analysis-Sanger sequencing combined procedure, mutational load
PolyPhen-2 and Mutation Taster software, and the frequency of mutations/variations was determined in the selected samples. The evaluation of mutational load revealed that the majority of the G3 EEC samples exhibited PIK3CA mutations (39\%) and PTEN mutations (67\%), and the majority of the samples $(83 \%)$ had mutations in at least one of the two genes, and $33 \%$ had mutations in the two genes. The results of the present pilot study suggested that the cost-effective combined PCR-HRMA and Sanger sequencing procedure may be suitable for identification of PTEN and PIK3CA mutations in G3 EEC and that their frequency was consistent in G3 EEC, indicating that the PI3K pathway serves a pivotal function that may have potential for defining targeted therapy for the treatment of G3 EEC.

\section{Introduction}

Endometrial cancer (EC) comprises a biologically and clinically heterogeneous group of tumors, generally classified as Type I (endometrioid) or Type II (non-endometrioid) on the basis of morphological characteristics. It was recently demonstrated that these two groups are characterized by different genetic alterations; consequently, the function of these variations has been widely investigated, in order to identify diagnostic and prognostic markers for improving the delineation of EC (1).

Recently, a novel classification system was published by The Cancer Genome Atlas (TCGA) on the basis of genetic and epigenetic modifications (2). In addition, a previous TCGA-based analysis has confirmed that $>90 \%$ of endometrioid EC (EEC) harbor genetic alterations in the phosphoinositide 3-kinase (PI3K) signaling pathway (3), primarily concerning PI3K catalytic subunit $\alpha$ (PIK3CA), and phosphatase and tensin homolog (PTEN) (3-12).

Furthermore, it has been identified that grade 3 (G3) EEC exhibits clinical and pathological behavior between type I and type II, suggesting that EEC may evolve by mechanisms different from those for other EEC grades (13-16).

In EEC, the frequency of PIK3CA mutations is $50 \%$ (17) making it one of the most frequently mutated genes (18-21), independently of histological types $(17,22)$. In total, $>70 \%$ of PIK3CA mutations cluster at three 'hotspot' codons, exon 1 
(p.R88Q), exon 9 (p.E542K, p.E545K/G/A, p.Q546R) and exon 21 (p.H1047R/L/Y, p.M1043I/V) (17,23), each of which induces its constitutive activation (3,24-26). However, the association between genetic variations and endometrial carcinogenesis remains poorly understood. Several studies have identified different correlations between PIK3CA variations and tumor grade, stage and type (5,6,26-29).

PTEN somatic mutations have been reported in several types of gynecological tumor; in addition, PTEN inactivation is present in endometriosis and endometrial hyperplasia, and may be considered an early event in cancer development (30-33). In EECs, PTEN mutations have been identified (16), and PTEN protein loss was revealed to be correlated with overall survival (OS) (34).

As suggested by TCGA, the PI3K signaling pathway may represent a potential target for targeted therapy for the treatment of EC $(3,23)$. Indeed, several PI3K pathway inhibitors have been developed and are currently under investigation in preclinical studies, and in early clinical trials (35), with various results depending on the therapeutic agent used and treatment set-up $(8,18,20,22,24,26,29,36-44)$. Nevertheless, it is currently unclear how PIK3CA and PTEN mutations affect the sensitivity to inhibitors in EC (45-47). Consequently, efforts should be focused on improving the characterization of the $P I K 3 C A$ and PTEN mutational profiles, to allow the establishment of personalized targeted therapy.

Several approaches may be used for the characterization of mutations [e.g. next-generation sequencing (NGS), mass spectrometry (MS), Sanger sequencing, polymerase chain reaction (PCR)-high-resolution melting analysis (HRMA) and quantitative PCR (qPCR)]. Certain methods (i.e. NGS and MS) provide a comprehensive overall evaluation with high specificity and sensitivity, but require expensive instrumentation and procedures, and trained personnel; other methods (i.e. Sanger sequencing, PCR-HRMA and qPCR) are simpler and more cost-effective, but allow the analysis of only a limited number of selected exons, and the specificity and sensitivity differ (Sanger sequencing had high specificity and low sensitivity, and vice versa for PCR-HRMA, whereas qPCR is sensitive and specific, but allows the analysis of only single known hotspots) (48-51).

The present pilot study had two main aims: i) To develop an economical and efficient combined procedure on the basis of PCR-HRMA and Sanger sequencing for evaluating the presence of variants in PIK3CA (exons 1, 9 and 21) and PTEN (exons 5, 6, 7 and 8) genes with the intention to overcome the limitations of each technique; and ii) to assess the presence of the primary mutations in PIK3CA and PTEN in a selected homogeneous subgroup of G3 EECs.

\section{Materials and methods}

Tissue collection and DNA extraction. A total of 18 available and suitable tissues were selected from patients with G3 EEC and with at least 2 years of follow up (up to 1 February 2018). All patients underwent surgical treatment between May 2013 and November 2015, at the Obstetrics and Gynecology Unit, Careggi University Hospital (Florence, Italy). Patients were treated depending on International Federation of Gynecology and Obstetrics (FIGO staging), grading and European Society of Gynecological Oncology (ESGO) risk (52). Tumor samples were collected at the time of surgery, following acquisition written informed consent from the patients. The study was approved by the Ethics Committee of Careggi University Hospital. Patients' demographic, clinical and pathological features are presented in Table I.

Tumor samples were immediately fixed in formalin and processed for paraffin embedding. The resulting formalin-fixed paraffin-embedded (FFPE) tissues were examined histologically by pathologists at Careggi University Hospital for the selection of areas containing $>70 \%$ of tumor cells.

DNA was extracted from slices using a QIAamp DNA FFPE tissue kit (Qiagen GmbH, Hilden, Germany), according to the manufacturer's protocol. DNA quality (ratio R) and quantity (DNA concentration) were determined using a spectrophotometer (NanoDrop ${ }^{\circledR} 1000$ UV spectrophotometer; Thermo Fisher Scientific, Inc., Waltham, MA, USA).

Primers and PCR conditions. Primers for selected exons of PIK3CA and PTEN were custom-made by Sigma-Aldrich; Merck KGaA (Darmstadt, Germany), and were designed to be suitable for Sanger sequencing and PCR-HRMA using primer3web software (version 4.1.0; primer3.ut.ee), checked for specificity and mismatch using Primer-BLAST (www. ncbi.nlm.nih.gov/tools/primer-blast) and using SNP Check (version 3; secure.ngrl.org.uk/SNPCheck/snpcheck.htm). Primer sequences are presented in Table II.

PCR was performed using the custom primers, Taq PCR core kit (Qiagen $\mathrm{GmbH}$ ) and the third-generation DNA-intercalating dye Syto 9 (Thermo Fisher Scientific, Inc.). Briefly, 10 ng DNA was amplified using $2 \mu 1$ master mix (10X), $0.5 \mu \mathrm{l}$ each primer $(10 \mu \mathrm{M}), 0.4 \mu \mathrm{l} \mathrm{dNTPs}$ (10 mM each), $0.4 \mu \mathrm{l}$ Syto9 $(50 \mu \mathrm{M}), 0.1 \mu \mathrm{l}$ Taq DNA polymerase $(5 \mathrm{U} / \mu \mathrm{l})$ and water in a final volume of $20 \mu \mathrm{l}$. Samples were subjected to incubation at $95^{\circ} \mathrm{C}$ for $5 \mathrm{~min}$, then 40 amplification cycles of $95^{\circ} \mathrm{C}$ for $30 \mathrm{sec}, 56 / 60^{\circ} \mathrm{C}\left(56^{\circ} \mathrm{C}\right.$ for $P T E N$ and $60^{\circ} \mathrm{C}$ for $\left.P I K 3 C A\right)$ for $45 \mathrm{sec}$ and $72^{\circ} \mathrm{C}$ for $45 \mathrm{sec}$, and a final incubation at $72^{\circ} \mathrm{C}$ for $20 \mathrm{~min}$ in a GeneAmp PCR system 9700 (Thermo Fisher Scientific, Inc.).

HRMA. Amplified samples were submitted to a high-resolution melting (HRM) protocol $\left(95^{\circ} \mathrm{C}\right.$ for $5 \mathrm{~min}, 40^{\circ} \mathrm{C}$ for $1 \mathrm{~min}$, ramping temperature from 72 to $84^{\circ} \mathrm{C}$ ) in a Rotor-gene Q PCR cycler (Qiagen $\mathrm{GmbH}$ ). The melting curve of each sample was analyzed using melting and HRM software (Rotor-Gene ${ }^{\circledR}$ Q-Pure Detection series software, version 2.1.0, build 9; Qiagen $\mathrm{GmbH}$ ).

Sanger sequencing. The amplified and melted PCR products were purified using a QIAquick PCR purification kit (Qiagen $\mathrm{GmbH}$ ) and sequenced on an ABI 310 genetic analyzer (Thermo Fisher Scientific, Inc.). Briefly, $3 \mu 1$ purified PCR was added to $1 \mu \mathrm{l}$ BigDye Terminator, $3.2 \mu \mathrm{l}$ forward/reverse primer $(1 \mu \mathrm{M}), 3.5 \mu \mathrm{l}$ BigDye Terminator buffer and water in a final volume of $20 \mu \mathrm{l}$, and amplified for 25 cycles of $96^{\circ} \mathrm{C}$ for $10 \mathrm{sec}, 59^{\circ} \mathrm{C}$ for $5 \mathrm{sec}$ and $60^{\circ} \mathrm{C}$ for $2 \mathrm{~min}$ in the GeneAmp PCR system 9700, purified using a DyeEx 2.0 Spin column (Qiagen $\mathrm{GmbH}$ ), according to the manufacturer's protocol, and $7 \mu \mathrm{l}$ was Sanger sequenced. 


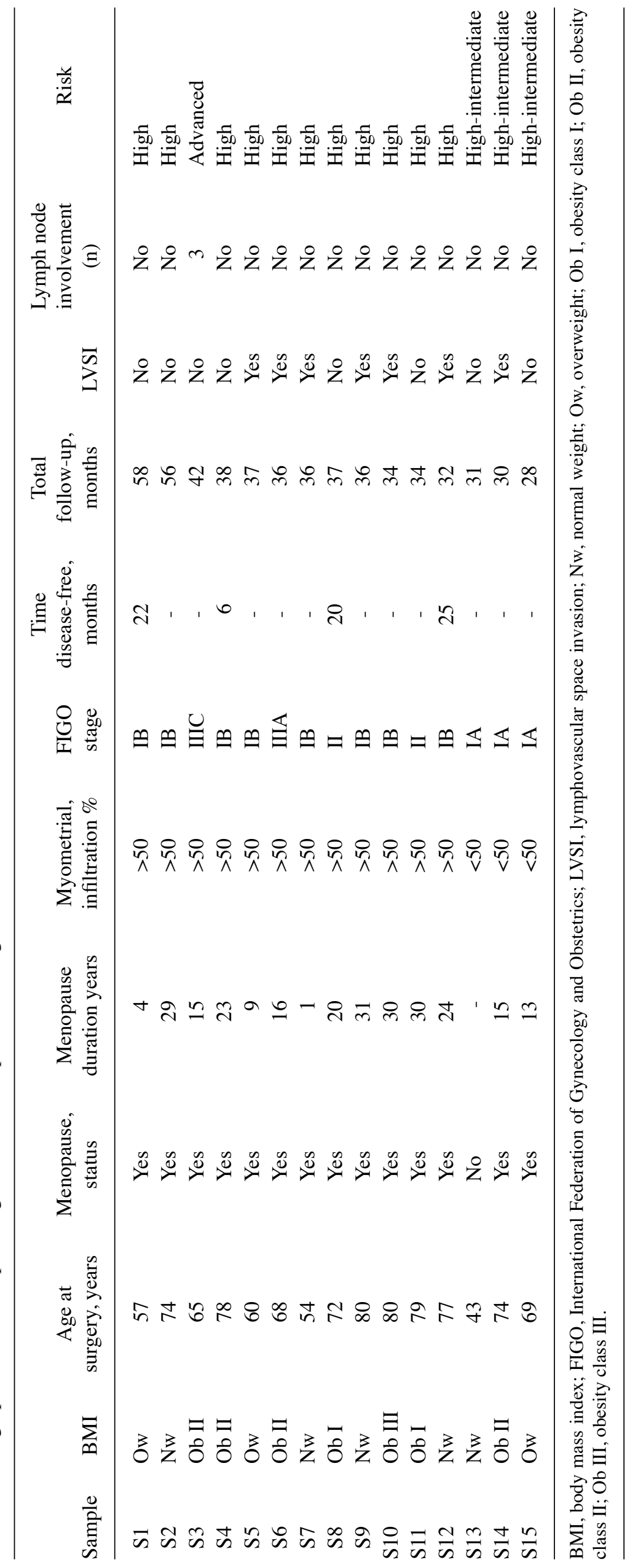


Table II. Primer sequences.

\begin{tabular}{|c|c|c|c|c|}
\hline Gene & Exon & Forward primer & Reverse primer & Product size, bp \\
\hline \multirow[t]{3}{*}{ PIK3CA } & 1 & 5'-TGTTACTCAAGAAGCAGAAAGGG-3' & 5'-ACGAAGGTATTGGTTTAGACAGA-3' & 231 \\
\hline & 9 & 5'-AGGGAAAATGACAAAGAACA-3' & 5'-ACCTGTGACTCCATAGAAA-3' & 124 \\
\hline & 21 & 5'-TGCTCCAAACTGACCAAACTG-3' & 5'-TGCATGCTGTTTAATTGTGTGG-3' & 299 \\
\hline \multirow[t]{4}{*}{ PTEN } & 5 & 5'-TGTGAAGATCTTGACCAATGGC-3' & 5'-AAATTCTCAGATCCAGGAAGAGG-3' & 231 \\
\hline & 6 & 5'-ACGACCCAGTTACCATAGCA-3' & 5'-TGTGAAACAACAGTGCCACT-3' & 185 \\
\hline & 7 & 5'-CCTCAGTTTGTGGTCTGCC-3' & 5'-GCCAGAGTAAGCAAAACACCT-3' & 296 \\
\hline & 8 & 5'-TACCAGGACCAGAGGAAACC-3' & 5'-AGCAAGTTCTTCATCAGCTGT-3' & 280 \\
\hline
\end{tabular}

To confirm identified mutations, the samples were also sequenced using reverse primers. The electropherogram of each sequenced amplicon was independently evaluated by two individuals. For validation of the combined procedure, in a subgroup of three samples, NGS was performed using Ion Torrent S5 with HotSpot cancer panel version 1 on Chip 520 (Thermo Fisher Scientific, Inc.) with a coverage (mean depth) average of 1,000X, mean reads of 400,000 and mean read length of $115 \mathrm{bp}$, according to the manufacturer's protocol.

For validation of variations identified by PCR-HRMA, but not by Sanger sequencing (sample S2 and S4), their corresponding PCR products associated with PTEN exon 5 were cloned using TOPO-TA cloning (Thermo Fisher Scientific, Inc.), according to the manufacturer's protocol. A total of 20 colonies was isolated and the plasmids were extracted using a QIAprep Spin Miniprep kit (Qiagen $\mathrm{GmbH}$ ). The extracted plasmids were sequenced as aforementioned.

Mutation identification. The nucleotide changes were evaluated using the Catalogue of Somatic Mutations in Cancer (COSMIC) database (version 86; cancer.sanger. ac.uk/cosmic), ensembl (release 93; www.ensembl.org/index. html), PolyPhen-2 (version 2; genetics.bwh.harvard.edu/pph2) and Mutation Taster (MT) (version 2; current build, NCBI 37/Ensembl 69; www.mutationtaster.org) software. Single nucleotide polymorphisms (SNPs) were confirmed using UCSC Genome Browser (genome.ucsc.edu/). The clinical impact was evaluated using COSMIC by Functional Analysis Through Hidden Markov Models score (pathogenic score, range: 0 , not pathogenic, to 1 , pathogenic), for single amino acid substitutions by PolyPhen-2 score (range: 0, not pathogenic, to 1 , pathogenic) and for synonymous single amino acid substitutions, complex variations (insertions/deletions/frameshifts) and modifications in intronic sequence by MT [evaluation: Disease-causing (DC) or SNP].

\section{Results}

Quality of DNA. The extracted DNAs had sufficient amount and quality for PCR amplification and Sanger sequencing; in particular, the concentration mean was $164.15 \pm 2.00 \mathrm{ng} / \mu \mathrm{l}$; the median was $138.00 \pm 2.00 \mathrm{ng} / \mu \mathrm{l}$; the range was between 18.00 and $299.10 \mathrm{ng} / \mu \mathrm{l}$; the value of $\mathrm{R}$ was 2.15 (mean) and 2.15 (median), and the range was between 2.00 and 2.47.
Establishment of the combined PCR-HRMA and Sanger sequencing procedure. The combined procedure is based on the sequential execution of PCR-HRMA and Sanger sequencing, without other steps, and avoids double PCR if the two procedures are performed separately with a corresponding saving of DNA, time of execution and limiting the number of mistakes due to performing the analysis twice. All samples were amplified to verify the success of amplification and to perform the HRMA. An appropriate curve was obtained for all the samples and for each analyzed exon. The HRMA suggested the presence of nucleotide alteration(s) without providing direct information on the specific nucleotide change(s), which induced changes in the shape of the HRM and melting curves. In particular, the presence of nucleotide change(s) induced or a shift in the peak or the appearance of additional peak(s) in the melting curve and a change in the shape of the HRM curve. In order to identify associations of the curve shape with a specific nucleotide sequence, the amplicons were sequenced using the Sanger procedure.

Figs. 1 and 2 present representative results of the HRM and melting curves, and the corresponding specific base modification identified by Sanger sequencing, for PIK3CA exon 1 and for PTEN exon 5, respectively.

The comparison of HRMA and sequencing data confirmed that samples with the same HRM curve shared the same nucleotide sequence. Differences in HRM curves for the same amplicon suggested the presence of different nucleotide sequences. Figs. 3 and 4 present the principal variations detected by HRM curves along with the corresponding nucleotide change(s) for each exon of PIK3CA and PTEN analyzed. Notably, for PTEN exon 5, it was observed that three samples shared the same HRM and melting curves (Fig. 5), but the corresponding Sanger sequences revealed the presence of a specific variant only for one sample (sample S9; p.Y155S), suggesting that the two samples (samples S2 and S4) may have the same variation as sample $S 9$, but at lower levels. To verify this hypothesis, the PCR products of samples S2 and S4 were cloned into a plasmid and sequenced, which confirmed the presence of the suggested variant at low level (two and three positive colonies, respectively, in samples S2 and S4).

This combined approach allowed the avoidance of a double PCR (if the two methodologies were applied separately), retaining DNA for use in further analysis. Furthermore, the use of HRMA alone suggested the presence of homoduplex 
A

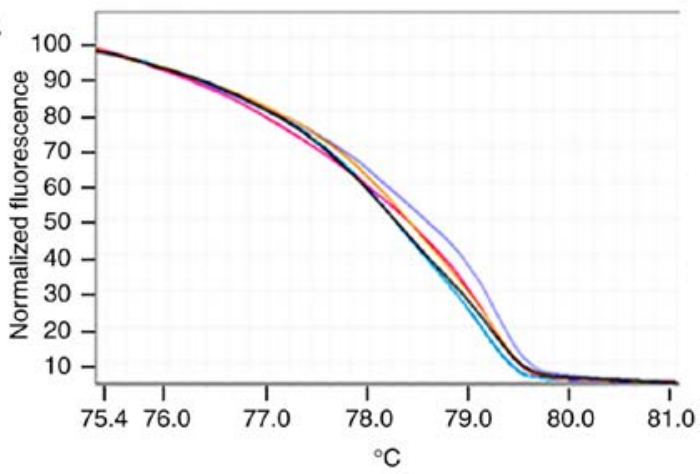

IVS1+40A>G (A/G) $+C .262 C>G(C / G)(S 11)$

c.266T>G (T/G) (S10)

$\mathrm{c} .262 \mathrm{C}>\mathrm{G}(\mathrm{C} / \mathrm{G})(\mathrm{S} 2)$

IVS1+40A>G (A/G) (S5)

Wt-wild type (amplicon sequence without variations) (S3)

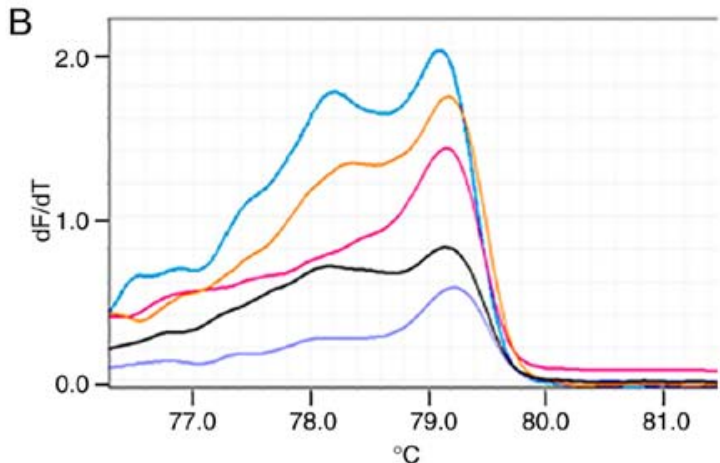

C

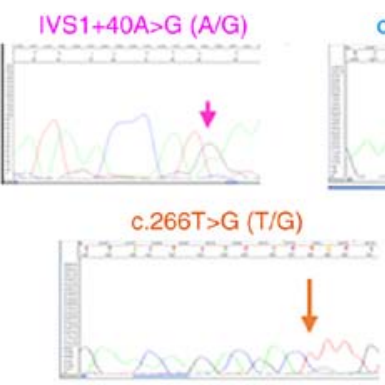

Figure 1. Investigation of the principal genetic variants in phosphoinositide 3-kinase catalytic subunit $\alpha$ exon 1. Each curve represents an exemplificative sample. The same color is used to represent the sample and the corresponding identified variation(s); 'wt' corresponds to a sample(s) that presented no variation in the specific amplicon in comparison with the GenBank reference sequence. (A) High-resolution melting curves. (B) Melting curves (C) Sanger hotspot sequence. If the mutation was present in more than one sample, only one sequence is presented. wt, wild-type.
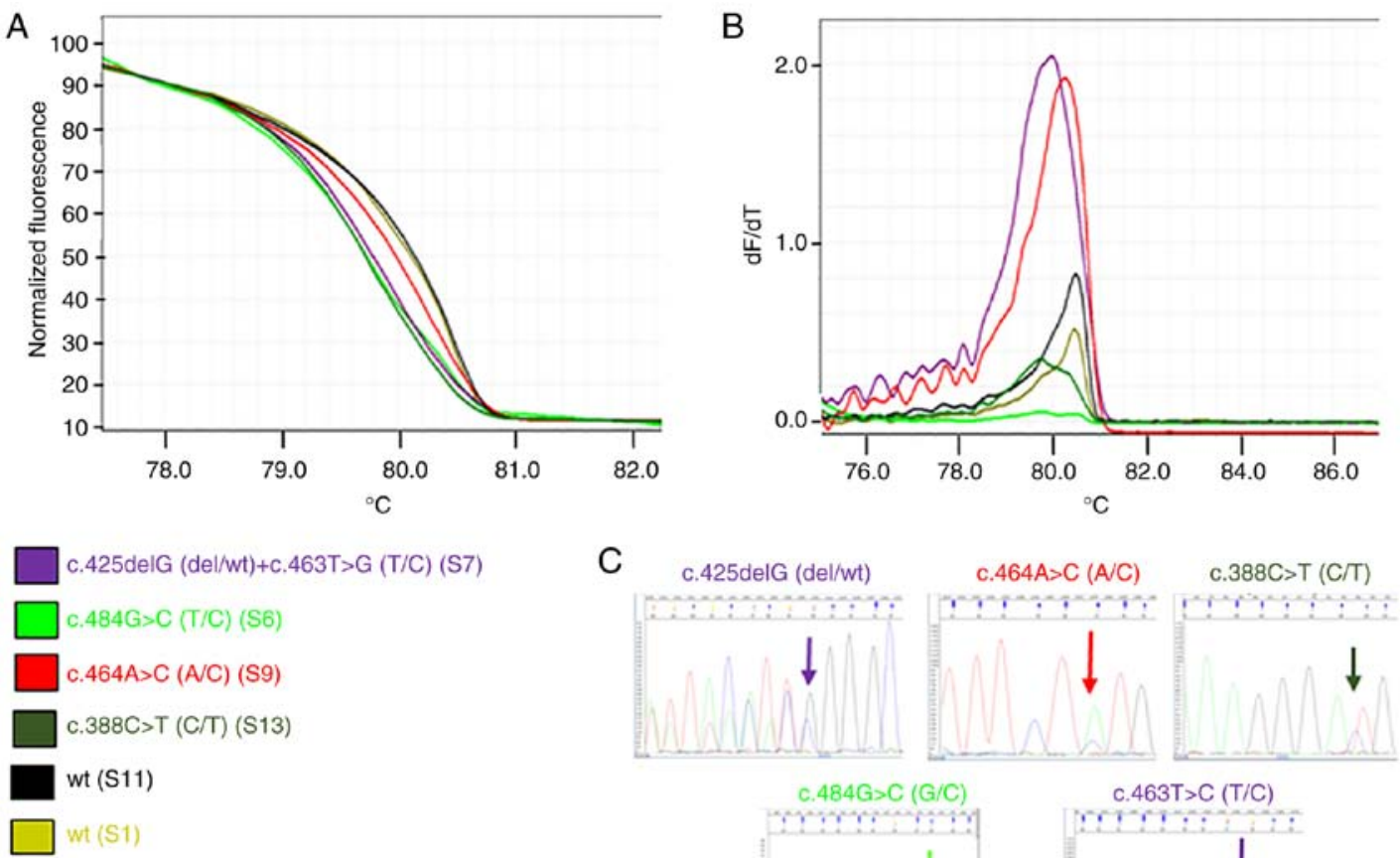

Wt: wild-type (amplicon sequence without variations)

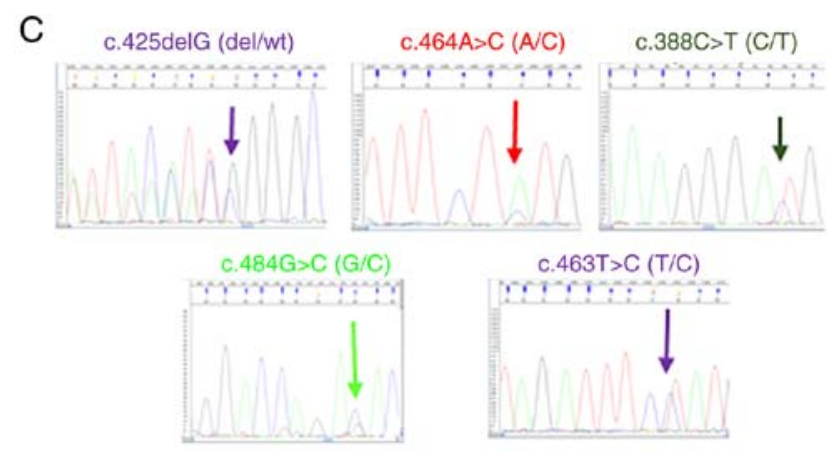

Figure 2. Investigation of the principal genetic variants in phosphatase and tensin homolog exon 5. Each curve represents an exemplificative sample. The same color is used to represent the sample and the corresponding identified variation(s); 'wt' corresponds to a sample(s) that presented no variation in the specific amplicon in comparison with the GenBank reference sequence. (A) High-resolution melting curves. (B) Melting curves. (C) Sanger hotspot sequence. If the mutation was present in more than one sample, only one sequence is presented. wt, wild-type.

or heteroduplex variations in the analyzed exons $[P I K 3 C A$ exon $1(61 \%)$, exon $9(6 \%)$ and exon $21(33 \%)$, and PTEN exon $5(44 \%)$, exon $6(78 \%)$, exon $8(50 \%)$ and none in exon 7$)$, but provided information on the type of variation. Sanger sequencing provided this specific information on the type of alteration, allowing the characterization of the nucleotide 
A

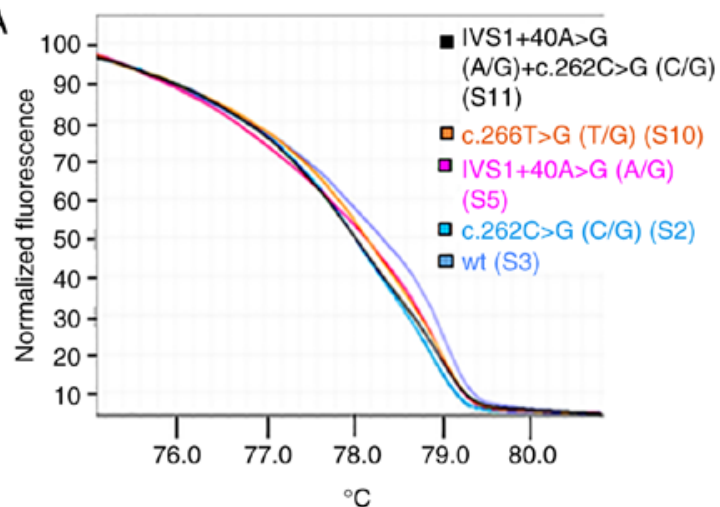

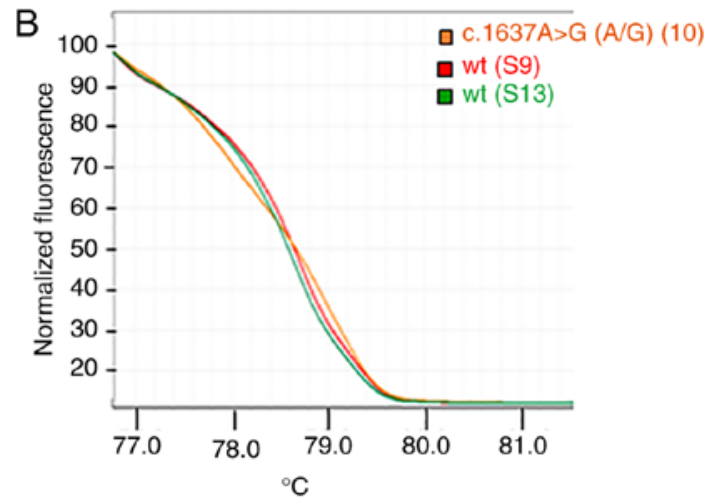

Wt, wild-type (amplicon sequence without variations)

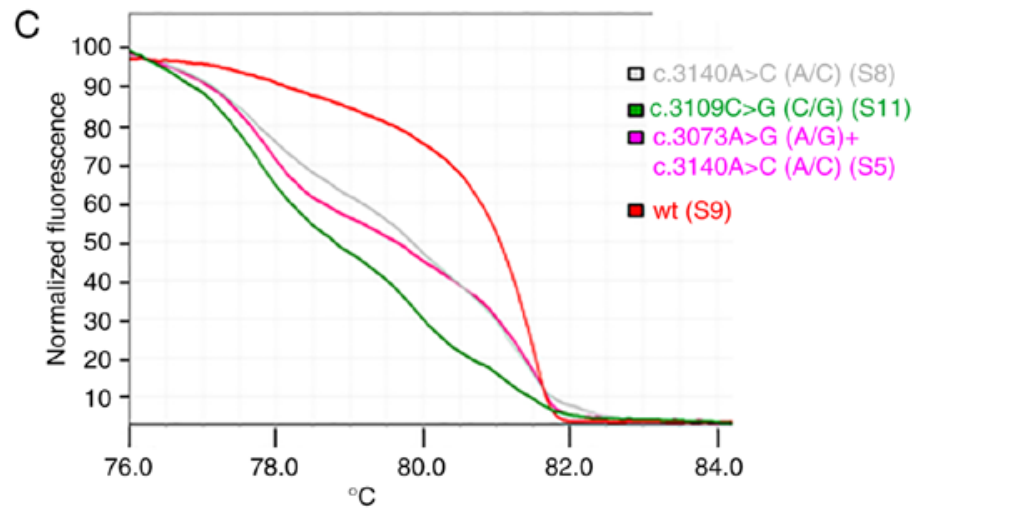

Figure 3. High-resolution melting curves associated with to the principal identified phosphoinositide 3-kinase catalytic subunit $\alpha$ gene variations. Each curve represents an exemplificative sample. The color key indicates the sample and the corresponding principal variation identified. The term 'wt' corresponds to a sample(s) that presented no variation in the specific amplicon in comparison with the GenBank reference sequence. (A) Exon 1. (B) Exon 9. (C) Exon 21. For each sample, the corresponding identified variants are presented in Table III. wt, wild-type.

variation, but with low sensitivity. The merged information provided the sensitivity of HRMA and the specificity of Sanger sequencing (for example, for PTEN exon 5, the mutation detection frequency increased from 33 to $44 \%$ ).

To further evaluate this approach, three samples were validated using this combined procedure and by NGS technology in which all the PTEN and PIK3CA exons were analyzed, as well as 48 other cancer-associated genes. The two technologies yielded the same results: For sample S16 only, a single-base mutation in PTEN exon 5 was detected.

\section{Evaluation of variants on PIK3CA and PTEN.}

$P I K 3 C A$. A total of seven different variations in 11 samples (61\%) were identified, all of which were heterozygous, except for IVS1+40A > G (intronic) which in three samples was homozygous and 7/11 were pathogenic (Table III). In particular, in exon 1, two mutations were identified, namely p.L89R (6\%) and p.R88G (18\%), as well as the SNP IVS1+40A >G (rs3729674; 44\%); in exon 9, the mutation p.Q546R (cosm12459; 6\%), was identified; and in exon 21, the mutation p.H1047P (cosm249874; $22 \%)$ and the variant p.E1037Q (12\%) were detected. The highest number of mutations (three mutations and one variant) was observed in exon 21 compared with exon 1 (two mutations) and exon 9 (one mutation). All five samples at FIGO stage III (IIIA and IIIC) did not exhibit any variation in PIK3CA.

PTEN. A total of 12 different variations were identified and $16 / 18(89 \%)$ samples exhibited at least one variation, among which $12(67 \%)$ presented a pathological variation (Table IV). In particular, exon 6 exhibited the highest number of variations. The most represented alteration was p.Q171Q (78\%), followed by p.R189K (cosm1745951; 44\%). Exon 5 exhibited pathogenic mutations in six samples (33\%): p.Y155S (18\%), p.D162H (cosm5274; 12\%), p.Y155H (cosm5038; 6\%), p.G143fs*4 (cosm30623; 6\%), R130* (cosm5152; 6\%) and G129Stop (cosm18663; 6\%). Exon 8 had an intronic variant (IVS8+32T $>$ G; 44\%) and in one sample it was associated with p.I300fs ${ }^{*} 2$. Exon 7 did not present any mutation according to Kafshooz et al (53). No particular trend was observed for the PTEN mutation, except for the non-menopausal patient that had PTEN R130* and the presence of the PTEN T167fs* in the obese class III patient.

Variant/mutational load. Regarding the overall variant evaluation, the majority of the samples (89\%) exhibited at least one variation (Table V). Regarding SNPs, 50\% of the samples exhibited one SNP and $18 \%$ presented with two SNPs.

Regarding the mutations, $67 \%$ of the samples had confirmed mutations at least in one of the two genes, whereas $18 \%$ had mutations in the two genes, and these values increased to 72 and $33 \%$, respectively, if the mutations predicted with PolyPhen-2 were considered, and to 82 and $39 \%$, respectively, if the MT prediction was considered.

It was observed that $28 \%$ of the samples exhibited a single confirmed mutation, $16 \%$ exhibited two mutations, $22 \%$ exhibited three mutations and $6 \%$ exhibited four mutations. Considering the overall variant load (excluding SNPs), $18 \%$ of 

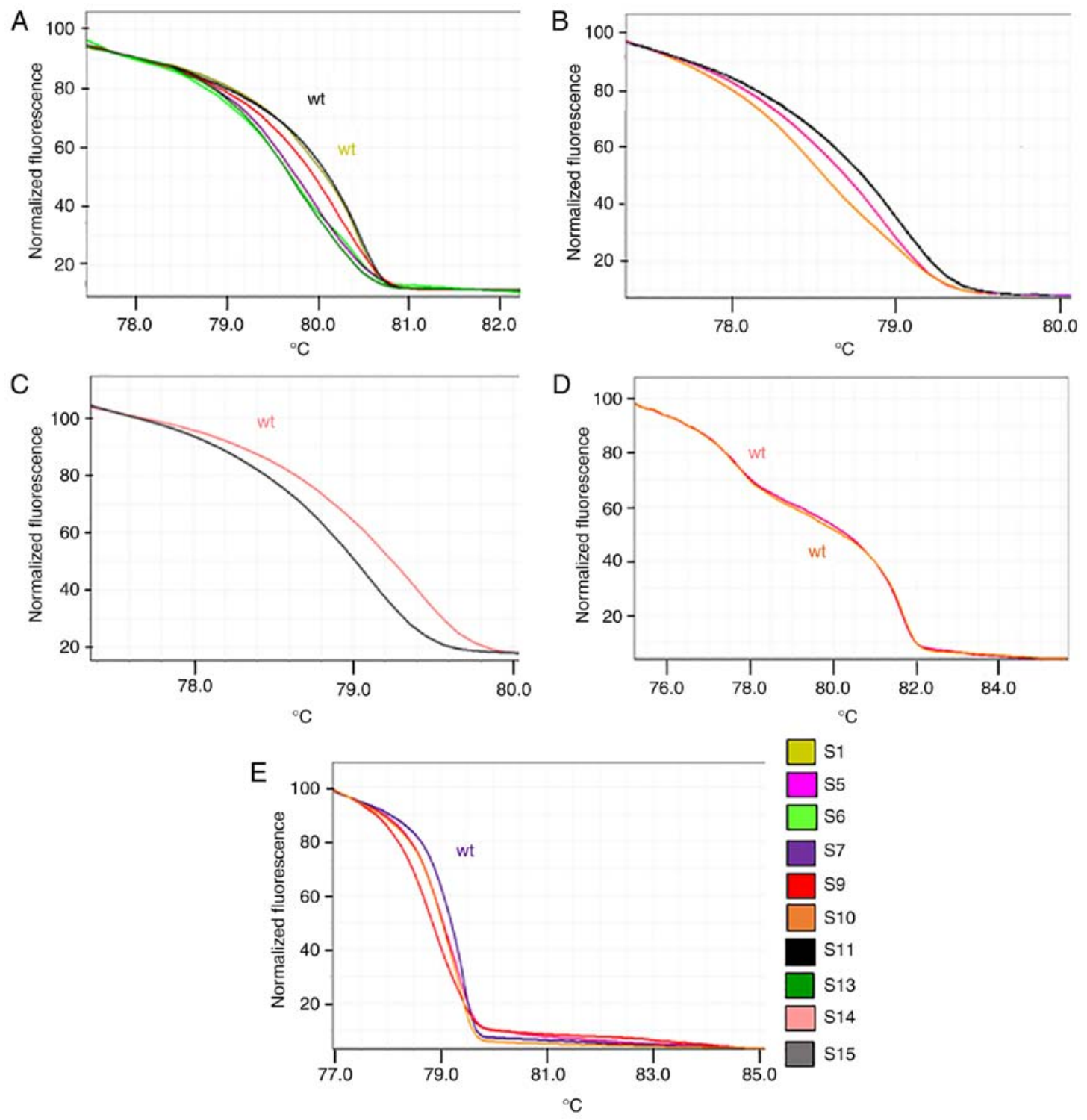

Figure 4. High-resolution melting curves associated with the principal identified phosphatase and tensin homolog gene variants. Each curve represents an exemplificative sample. 'wt' corresponds to a sample(s) that presented no variation in the specific amplicon in comparison with the GenBank reference sequence. (A) Exon 5. (B) Exon 6. (C) Exon 6. (D) Exon 7. (E) Exon 8. For each sample, the corresponding identified variants are presented in Table IV. wt, wild-type.
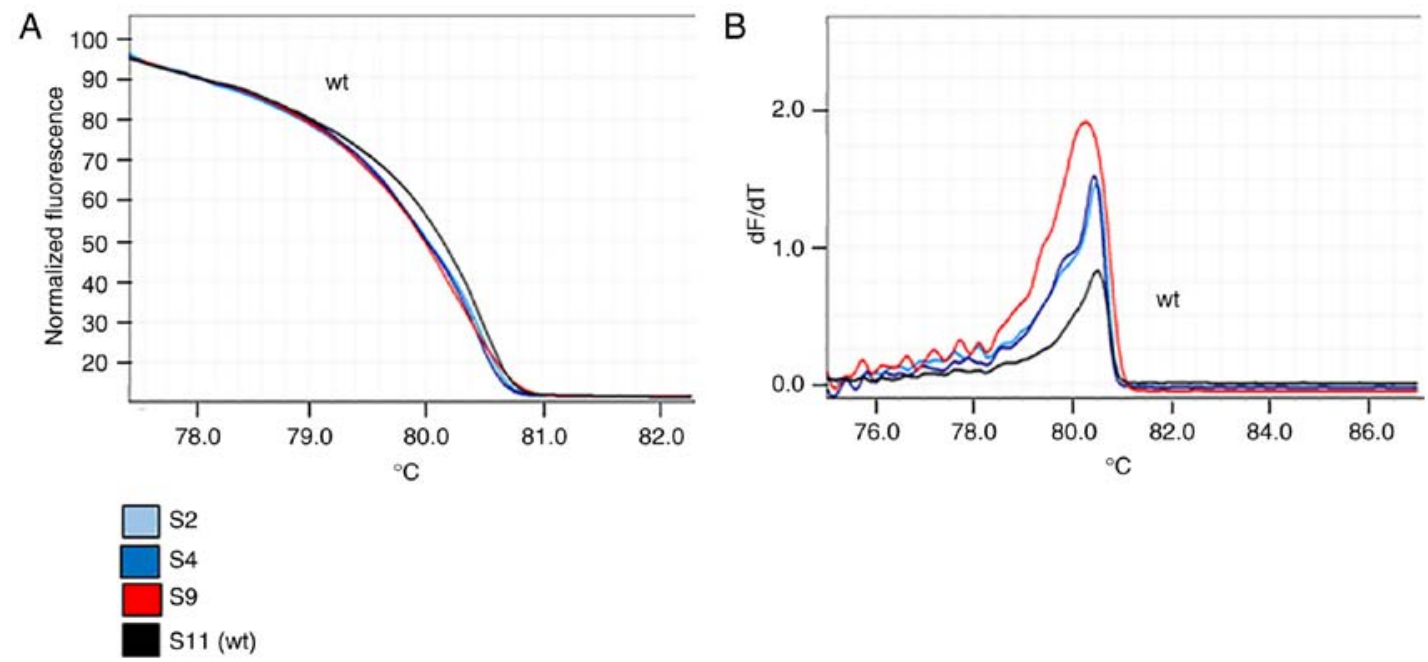

Figure 5. Investigation of phosphatase and tensin homolog exon 5 variant c.464A>C by HRM analysis in samples S9 (red), S2 (light blue) and S4 (blue). S11 represents the reference 'wt' (sample with no variations in this amplicon). (A) HRM curves. (B) Melting curves. HRM, high-resolution melting; wt, wild-type. 


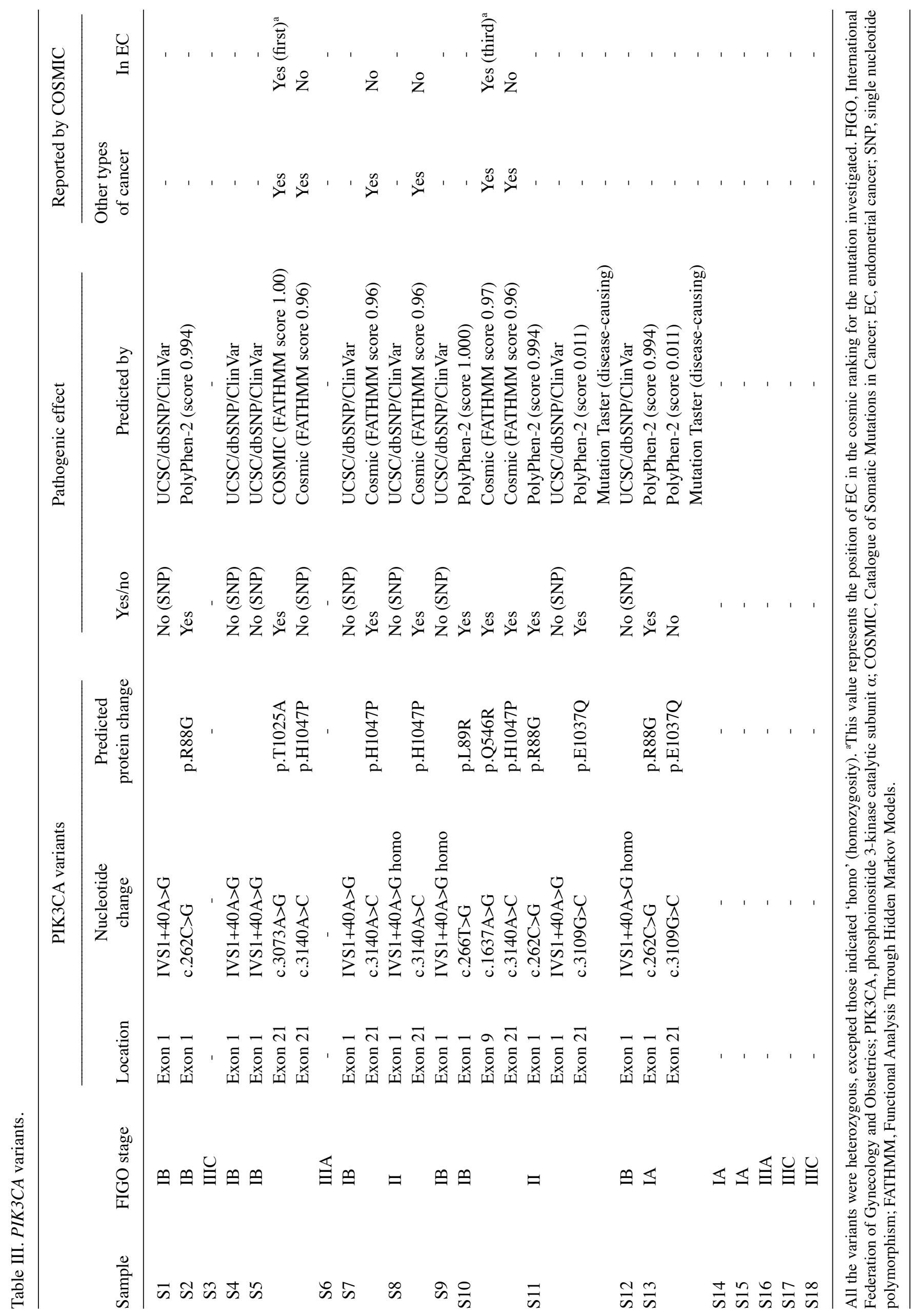




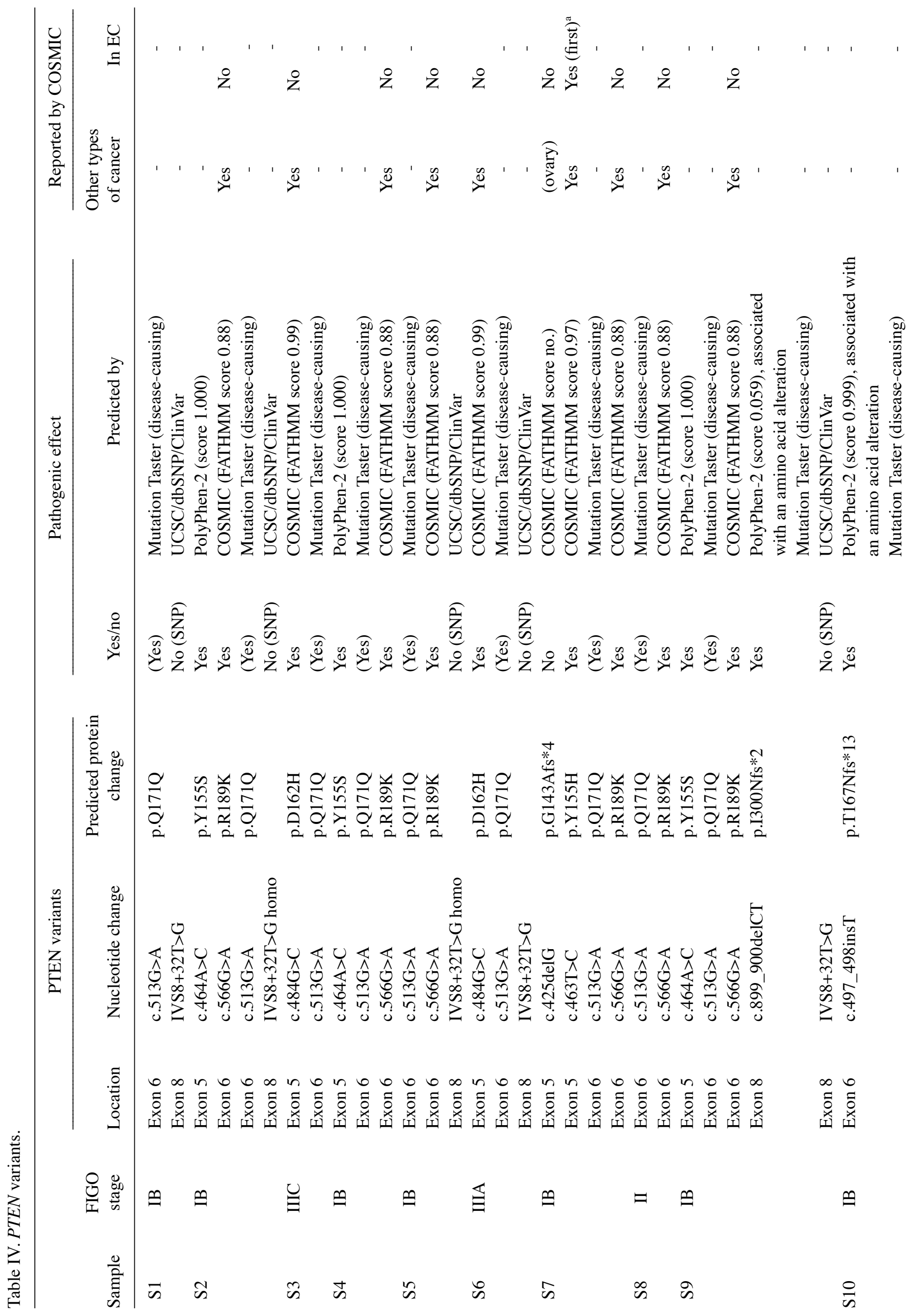




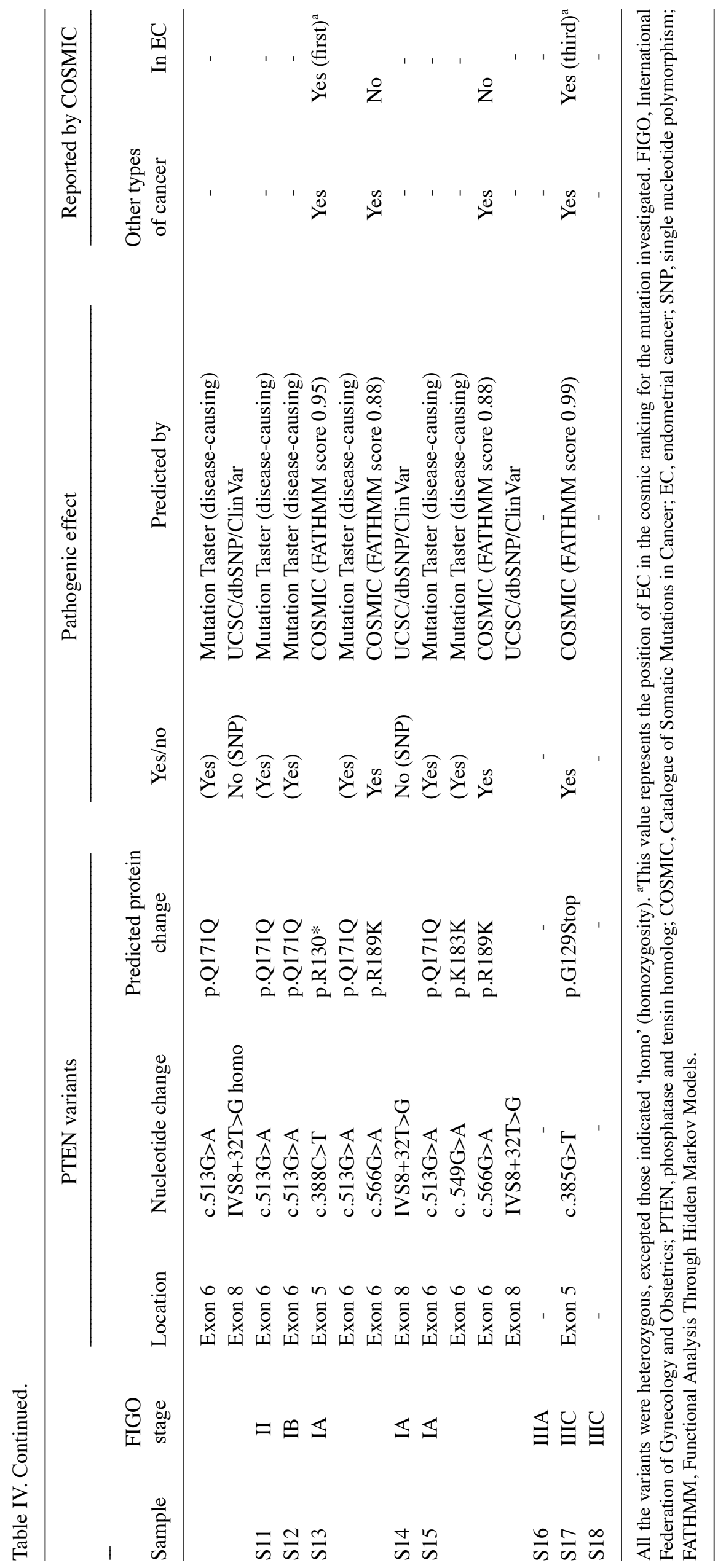


Table V. Overall evaluation of PIK3CA and PTEN variants in the samples analyzed.

\begin{tabular}{|c|c|c|}
\hline Sample & $P I K 3 C A$ variants & $P T E N$ variants \\
\hline $\mathrm{S} 1$ & $I V S 1+40 A>G$ & p.Q171Q, $I V S 8+32 T>G$ \\
\hline $\mathrm{S} 2$ & p.R88G & p.R189K, p.Y155S\$, p.Q171Q, $I V S 8+32 T>G$ \\
\hline S3 & & p.D162H, p.Q171Q \\
\hline S4 & $I V S 1+40 A>G$ & p.R189K, p.Y155S§, p.Q171Q \\
\hline S5 & p.T1025A, p.H1047P, $I V S I+40 A>G$ & p.R189K, p.Q171Q, $I V S 8+32 T>G$ \\
\hline S6 & & p.D162H, p.Q171Q, $I V S 8+32 T>G$ \\
\hline S7 & p.H1047P, $I V S 1+40 A>G$ & p.Y155H, p.R189K, $p . G 143 A f s^{*} 4$, p.Q171Q \\
\hline S8 & p.H1047P, $I V S 1+40 A>G$ & p.R189K, p.Q171Q \\
\hline S9 & $I V S I+40 A>G$ & p.R189K, p.Q171Q, p.Y155S, $p . I 300 N f s^{*} 2, I V S 8+32 T>G$ \\
\hline S10 & p.Q546R, p.H1047P, p.L89R & p.T167Nfs $* 13$, p.Q171Q,$I V S 8+32 T>G$ \\
\hline S11 & p.R88G, p.E1037Q, $I V S 1+40 A>G$ & p.Q171Q \\
\hline S12 & $I V S I+40 A>G$ & p.Q171Q \\
\hline S13 & p.R88G, p.E1037Q & p.R130*, p.R189K, p.Q171Q \\
\hline S14 & & $I V S 8+32 T>G$ \\
\hline $\mathrm{S} 15$ & & p.R189K, p.Q171Q, p.K183K, IVS8+32T>G \\
\hline \multicolumn{3}{|r|}{$\mathrm{c}$} \\
\hline S17 & & p.G129Stop \\
\hline S18 & & \\
\hline
\end{tabular}

Italic represents single nucleotide polymorphisms. Bold represents variations predicted as 'pathogenic' using PolyPhen-2. Underlining represents variation predicted as 'pathogenic' using Mutation Taster. Bold and underlining represents variation confirmed as 'pathogenic' by Catalogue of Somatic Mutations in Cancer. PIK3CA, phosphoinositide 3-kinase catalytic subunit $\alpha$; PTEN, phosphatase and tensin homolog.

the samples exhibited none, one or four mutations, $10 \%$ exhibited two or five mutations, and $26 \%$ exhibited three mutations.

No specific mutation/variant load distribution was observed according to clinicopathological characteristics.

\section{Discussion}

Currently, there is increasing interest in investigating the genome alterations in tumors, with such research made possible by the development of specific and sensitive technologies (e.g. NGS or MassArray). These promising technologies are useful for understanding the mechanisms underlying carcinogenesis and cancer evolution, but are costly and require computational support for data analysis. For clinical application, the identification of genetic variations must be performed rapidly and cost-effectively, as usually performed by Sanger sequencing/pyrosequencing or PCR-HRMA procedures. Typically, qPCR is performed for confirmation analysis.

It is established that PCR-HRMA has a lower cost and is an accurate tool for mutation scanning, allowing the identification of variations through comparison of HRM curves; consequently, it is used as screening method or for the detection of known hotspot mutations. Its ability in discriminating known mutations is due to the use of reference material, or if this material is not available or novel variations are present in the samples analyzed, the technique is not able to identify the nucleotide change, but only to detect the presence (54-56). The HRMA curve shape depends on the number and type of nucleotide changes, with different nucleotide changes yielding different curves. The procedure alone is highly sensitive (overall sensitivity 0.1-5\%) $(50,51)$, but lacks specificity, since it does not allow for the direct evaluation of the specific nucleotide changes. Consequently, for the identification of nucleotide variations, the procedure requires the use of reference materials with the same alteration to be investigated along with the target samples, or further investigations by other techniques (such as sequencing or qPCR).

Sanger sequencing is the reference procedure for the identification of nucleotide changes for clinical purposes; even if this technology is unable to reveal variations at low levels (overall sensitivity $20 \%$ ) $(48,49)$, sometimes pyrosequencing is used due its increased sensitivity. However, this methodology is relatively expensive compared with Sanger sequencing, even if more sensitive (57).

Currently, other high-throughput methodologies are beginning to be used in the clinic for the identification of genetic variation in clinical samples, such as NGS and MS that allow the evaluation of multiple genes/exons and several samples in the same run. Usually these procedures improve specificity and sensitivity, but are expensive either for their required instrumentation and methodological procedures, provide abundant information that is not useful for clinical purposes, and require trained personnel for data analysis. In particular, MS allows the evaluation of known variations on specific exons/genes; instead NGS allows the evaluation of genes or genome and the identifications of new genetic variants. qPCR, which has high sensitivity and specificity, allows the identification of well-known and characterized variations, but is expensive and 
Table VI. Principal characteristics of the main procedures used for identification of variants in DNA sequence.

Procedure

\begin{tabular}{|c|c|c|c|c|c|c|c|}
\hline & \multicolumn{7}{|c|}{ Procedure } \\
\hline & \multicolumn{2}{|c|}{ High-throughput } & \multicolumn{5}{|c|}{ Single amplicon } \\
\hline & MassArray & NGS & qPCR & PCR-HRMA & $\begin{array}{c}\text { Sanger } \\
\text { sequencing }\end{array}$ & Pyrosequencing & $\begin{array}{c}\text { PCR-HRMA+ } \\
\text { Sanger sequencing }\end{array}$ \\
\hline Cost per sample & Medium & High & Medium & Low & Medium & Medium & Medium \\
\hline Sensitivity & High & High & High & High & Low & Medium & High \\
\hline Specificity & High & High & High & Low & High & High & High \\
\hline $\begin{array}{l}\text { Discovery of novel } \\
\text { variant }\end{array}$ & No & Yes & No & Yes & Yes & Yes/no ${ }^{a}$ & Yes \\
\hline $\begin{array}{l}\text { Identification of } \\
\text { sequence change }\end{array}$ & Yes & Yes & Yes & No & Yes & Yes & Yes \\
\hline $\begin{array}{l}\text { Multiple hotspot } \\
\text { within amplicon } \\
\text { length }\end{array}$ & $\begin{array}{l}\text { No in the } \\
\text { same well/ } \\
\text { yes in a run }\end{array}$ & Yes & No/yes ${ }^{b}$ & Yes & Yes & Yes & Yes \\
\hline $\begin{array}{l}\text { Multi-exon analysis in } \\
\text { the same preparation }\end{array}$ & Yes & Yes & No & No & No & No & No \\
\hline $\begin{array}{l}\text { Multi-sample analysis } \\
\text { in the same preparation }\end{array}$ & Yes & Yes & No & No & No & No & No \\
\hline Trained personnel & Yes & Yes & No & No & No & Medium & No \\
\hline Cost of instrumentation & High & High & $\begin{array}{l}\mathrm{No} / \\
\text { medium }^{\mathrm{c}}\end{array}$ & No & No & Medium & No \\
\hline Data analysis & Yes & Yes & No & No & No & No & No \\
\hline
\end{tabular}

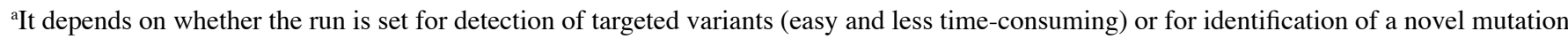
(more time-consuming and expensive compared with targeted approach). ${ }^{\mathrm{b}} \mathrm{It}$ depends on whether a multiplex or singleplex assay is used. ${ }^{\mathrm{c}} \mathrm{Sev}-$ eral instruments are present on the market with a broad range of prices. NGS, next-generation sequencing; PCR, polymerase chain reaction; qPCR, quantitative PCR; HRMA, high-resolution melting analysis.

time-consuming if several genes or variations require analysis, and is not suitable for the detection of unknown variations. It is typically used for confirmation when Sanger sensitivity fails.

The present study establishes a combined procedure that overcomes the limitations of each technique alone (low sensitivity of Sanger sequencing and low specificity of HRMA) and combines the strengths (high specificity of Sanger sequencing and high sensitivity of HRMA) of these techniques. It also allows a double check for the presence of variants in the main exons of PIK3CA and PTEN, in order to improve the reliability of the results. The target of this approach is the identification of variants in single samples and in amplicons of between 100 and $200 \mathrm{bp}$ in length; consequently, it cannot be compared with high-throughput procedures. Furthermore, this combined approach, in comparison with the application of either method alone, may decrease the amount of DNA used in the analysis (a single PCR is performed for the two methodologies) and the number of samples analyzed by Sanger sequencing. In particular, if a reference wild-type (wt) sample is used in the PCR-HRMA step, only the samples presenting HRM curves different from the wt should be analyzed by Sanger sequencing. Furthermore, PCR-HRMA can also only suggest the presence of novel variants, but they cannot be identified; by contrast, the combined use of PCR-HRMA and Sanger analysis can immediately confirm and identify the novel variations. This combined procedure may even be useful for defining patient eligibility for targeted therapy; it is known that targeted therapy is dependent on the identification of specific mutations by Sanger sequencing, and its efficacy is determined by the number of mutated cells and by the development of any new mutations that can originate from tumor heterogeneity or de novo. Owing to the high sensitivity of HRMA, suspected cases with a low proportion of mutations may still be identified and monitored (Table VI).

The present study focused on the analysis of specific exons of PIK3CA and PTEN, since these are established as the main genes involved in alterations in the PI3K signaling pathway, and they have been widely investigated for the characterization of EC as specific targets for personalized therapies on the basis of PI3K pathway inhibitors $(17,18,20,29,35,40)$. Indeed, early clinical data on several tumor types suggested that PIK $3 C A$ and PTEN mutations may affect the success of $\mathrm{PI} 3 \mathrm{~K} /$ protein kinase $\mathrm{B}(\mathrm{AKT}) /$ mammalian target of rapamycin (mTOR)-targeted therapies (58-61). Patients with gynecological tumors and PIK3CA mutations have demonstrated a $30 \%$ response rate $(\mathrm{RR})$ in early-phase clinical trials 
with PI3K/AKT/mTOR inhibitors, compared with a $10 \%$ $\mathrm{RR}$ in patients lacking PIK3CA mutations (42). It is conceivable that loss of PTEN function can be similarly predictive for therapy efficacy, whereas simultaneous mutations in the mitogen-activated protein kinase signaling pathway may lead to resistance to treatment $(58,60)$. Identifying actionable molecular aberrations has been a critical step for several major therapeutic advances in cancer medicine.

A combined technique was established in the present study for the examination of G3 EEC in order to investigate the involvement of major mutations of the PI3K signaling pathway in this EC subtype, and to ultimately implement the clinical and pathological knowledge of this grade of EEC regardless of differing characteristics (e.g. FIGO stage and ESGO risk). The present pilot study demonstrated that the approach was successful in analyzing G3 EEC, specifically for the genetic profile of selected PIK3CA and PTEN exons.

It was observed, in the cohort of the present study, that several samples were wt for specific exons (from $22 \%$ of PTEN exon 6 to $100 \%$ of PTEN exon 7) and that the overall number of variations was broad (in a single sample there were up to three variations for PTEN exon 6 and up to seven variations overall).

The focus was on the PIK3CA exons 1, 9, and 21, and on the PTEN exons 5, 6, 7 and 8 since these are typically the more frequently mutated exons in EECs; indeed, in a previous study, Rudd et al (17), when investigating all PIK3CA and PTEN exons, identified that no other exons were involved in G3 EEC. Furthermore, to verify this result and to partially validate the combined methodological approach, three samples were analyzed by NGS obtained the same results. Nevertheless, not examining all exons of PTEN and PIK3CA is a limitation of the present study, and perhaps something to perform in the future.

Using the approach developed in the present study, the overall evaluation revealed that $89 \%$ of samples exhibited at least one variation in either of the two genes, and that $83 \%$ had mutations. Regarding PIK $3 C A, 39 \%$ of samples exhibited mutations, among which the most frequent were H1047P (27\%), p.T1025A (7\%) and p.Q546R (7\%) $(7,17,21,22)$. With regard to PTEN, $67 \%$ of the samples had mutations, including p.R189K (53\%), p.D132H (13\%), p.Y155H (7/\%) and p.R130* (7\%).

As aforementioned, the overall mutational load was determined as at least one mutation in $83 \%$ of the samples and coexistence of mutations in the two genes in $33 \%$ of the samples. An increased mutation frequency was identified for each single gene compared with the coexistence of mutations, in contrast with results reported previously (27), particularly those reported by Oda et al (6), which may be due to the specific evaluation of the G3 subgroup of EEC in the present study.

It was observed that the FIGO stage III tumors did not exhibit any mutation in PIK3CA, and that the patient with class III obesity had PIK3CA p.Q546 and PTEN p.T167fs" mutations, suggesting an association supported by a previous study of the association of obesity and EC through the involvement of the PI3K signaling pathway (62).

The preliminary data of the present study indicated potential for the use of a combined technological approach for the identification of variants in PIK3CA and PTEN. Although they are preliminary results owing to the limited number of samples analyzed, the data confirm the involvement of PI3K pathway alterations in G3 EEC; however, further investigation is required.

In conclusion, the present study has demonstrated the suitability and reliability of a combined approach (PCR-HRMA and Sanger sequencing) for the evaluation of variants in selected exons of PIK3CA and PTEN in G3 EEC, suggesting that this approach may be useful for improving the classification and personalized treatment of patients with EC.

\section{Acknowledgements}

Not applicable.

\section{Funding}

The present study was supported by grants from the Tuscan Tumor Institute, Florence (Italy) and the University of Florence (Italy).

\section{Availability of data and materials}

The datasets used and/or analyzed during the current study are available from the corresponding author on reasonable request.

\section{Authors' contributions}

FM performed Sanger sequencing, evaluation of Sanger electropherograms, analyses and interpretation of HRMA and Sanger data, identification of nucleotide changes using databases (COSMIC, PolyPhen-2 and mutation taster), and was a major contributor in writing the manuscript. IT collected the clinical and pathophysiological data for each patient, evaluated the criteria for tissue collection and selection, and set up a database containing patient information. FS was involved in patient recruitment, collection of the clinical and pathophysiological data for each patient, and evaluation of the criteria for tissue collection and selection. EP performed the FFPE sample selection, the histological examination of selected FFPE slices, and the selection of appropriate slices for molecular analysis. FC performed the FFPE sample collection, FFPE slices and the histological examination of selected FFPE slices. MF performed the patient recruitment, the surgery and treatment on enrolled women, and collection of informed consent. FP co-ordinated the clinical team for patient recruitment and supervised the data analysis. SP performed DNA extraction and PCR, evaluation of Sanger electropherograms and contributed in editing of the manuscript. IN performed the patient recruitment, the surgery and treatment on enrolled women, the collection of informed consents, follow-up of patients and contributed in editing of the manuscript. All authors read, revised and approved the final manuscript.

\section{Ethics approval and consent to participate}

Research is performed on humans following international and national regulations in accordance with The Declaration of Helsinki, or any other relevant set of ethical principles. This 
research involved human subjects or tissues, and the authors state that informed consent for participation in the study or use of their tissue was obtained from all participants.

\section{Patient consent for publication}

The manuscript did not contain identifying information, including names, initials, date of birth, hospital numbers or images related to patients.

\section{Competing interests}

The authors declare that they have no competing interests.

\section{References}

1. Murali R, Soslow RA and Weigelt B: Classification of endometrial carcinoma: More than two types. Lancet Oncol 15: e268-e278, 2014.

2. Cancer Genome Atlas Research Network; Kandoth C, Schultz N, Cherniack AD, Akbani R, Liu Y, Shen H, Robertson AG, Pashtan I, Shen R, et al: Integrated genomic characterization of endometrial carcinoma. Nature 497: 67-73, 2013.

3. Marchiò C, De Filippo MR, Ng CK, Piscuoglio S, Soslow RA, Reis-Filh JS and Weigelt B: PIKing the type and pattern of PI3K pathway mutations in endometrioid endometrial carcinomas. Gynecol Oncol 137: 321-328, 2015.

4. McConechy MK, Ding J, Cheang MCU, Wiegand K, Senz J, Tone A, Yang W, Prentice L, Tse K, Zeng T, et al: Use of mutation profiles to refine the classification of endometrial carcinomas. J Pathol 228: 20-30, 2012.

5. Salvesen HB, Carter SL, Mannelqvist M, Dutt A, Getz G, Stefansson IM, Raeder MB, Sosh ML, Engelsen IB, Trovik J, et al: Integrated genomic profiling of endometrial carcinoma associates aggressive tumors with indicators of PI3 kinase activation. Proc Natl Acad Sci USA 106: 4834-4839, 2009.

6. Oda K, Stokoe D, Taketani Y and McCormick F: High frequency of coexistent mutations of PIK3CA and PTEN genes in endometrial carcinoma. Cancer Res 65: 10669-10673, 2005.

7. Catasus L, D'Angelo E, Pons C, Espinosa I and Prat J: Expression profiling of 22 genes involved in the PI3K-AKT pathway identifies two subgroups of high-grade endometrial carcinomas with different molecular alterations. Mod Path 23: 694-702, 2010.

8. Hayes MP, Wang H, Espinal-Witter R, Douglas W, Solomon GJ, Baker SJ and Ellenson HL: PIK3CA and PTEN mutations in uterine endometrioid carcinoma and complex atypical hyperplasia. Clin Cancer Res 12: 5932-5935, 2006.

9. van der Zee M, Jia Y, Wang Y, Heijmans-Antonissen C, Ewing PC, Franken P, DeMayo FJ, Lydon JP, Burger CW, Fodde $\mathrm{R}$, et al: Alterations in Wnt- $\beta$-catenin and Pten signalling play distinct roles in endometrial cancer initiation and progression. J Pathol 230: 48-58, 2013.

10. Sal V, Demirkiran F, Erenel H, Tokgozoglu N, Kahramanoglu I, Bese T, Turan H, Sofiyeva N, Calay Z, Arvas M and Guralp O: Expression of PTEN and $\beta$-catenin and their relationship with clinicopathological and prognostic factors in endometrioid type endometrial cancer. Int J Gynecol Cancer 26: 512-520, 2016.

11. McConechy MK, Ding J, Senz J, Yang W, Melnyk N, Tone AA, Prentice LM, Wiegand KC, McAlpine JN, Shah SP, et al: Ovarian and endometrial endometrioid carcinomas have distinct CTNNB1 and PTEN mutation profiles. Mod Pathol 27: 128-134, 2014.

12. Machin P, Catasus L, Pons C, Muñoz J, Matias-Guiu X and Prat J: CTNNB1 mutations and beta-catenin expression in endometrial carcinomas. Hum Pathol 33: 206-212, 2002.

13. Tejerizo-García A, Jiménez-López JS, Muñoz-González JL, Bartolomé-Sotillos S, Marqueta-Marqués L, López-González G and Gómez JF: Overall survival and disease-free survival in endometrial cancer: Prognostic factors in 276 patients. Onco Targets Ther 6: 1305-1313, 2013.

14. Mang C, Birkenmaier A, Cathomas G and Humburg J: Endometrioid endometrial adenocarcinoma: An increase of G3 cancers? Arch Gynecol Obstet 295: 1435-1440, 2017.
15. Voss MA, Ganesan R, Ludeman L, McCarthy K, Gornall R, Schaller G, Wei W and Sundar S: Should grade 3 endometrioid endometrial carcinoma be considered a type 2 cancer-a clinical and pathological evaluation. Gynecol Oncol 124: 15-20, 2012

16. Yasuda M: Immunohistochemical characterization of endometrial carcinomas: Endometrioid, serous and clear cell adenocarcinomas in association with genetic analysis. J Obstet Gyneacol Res 40: 2137-2176, 2014.

17. Rudd ML, Price JC, Fogoros S, Godwin AK, Sgroi DC, Merino MJ and Bell DW: A unique spectrum of somatic PIK3CA $(\mathrm{p} 110 \mathrm{~A} \alpha)$ mutations within primary endometrial carcinomas. Clin Cancer Res 17: 1331-1340, 2011.

18. Mackay HJ, Eisenhauer EA, Kamel-Reid S, Tsao M, Clarke B, Karakasis K, Werner HM, Trovik J, Akslen LA, Salvesen HB, et al: Molecular determinants of outcome with mammalian target of rapamycin inhibition in endometrial cancer. Cancer 15: 603-610, 2014

19. Mori N, Kyo S, Sakaguchi J, Mizumoto Y, Ohno S, Maida Y, Hashimoto M, Takakura M and Inoue M: Concomitant activation of AKT with extracellular-regulated kinase 1/2 occurs independently of PTEN or PIK3CA mutations in endometrial cancer and may be associated with favorable prognosis. Cancer Sci 98: 1881-1888, 2007.

20. Shoji K, Oda K, Kashiyama T, Ikeda Y, Nakagawa S, Sone K, Miyamoto Y, Hiraike H, Tanikawa M, Miyasaka A, et al: Genotype-dependent efficacy of a dual PI3K/mTOR inhibitor, NVP-BEZ235, and an mTOR inhibitor, RAD001, in endometrial carcinomas. PLoS One 7: e37431, 2012.

21. Millis SZ, Ikeda S, Reddy S, Gatalica Z and Kurzrock R: Landscape of phosphatidylinositol-3-kinase pathway alterations across 19784 diverse solid tumors. JAMA Oncol 2: 1565-1573, 2016.

22. Catasus L, Gallardo A, Cuatrecasas M and Prat J: Concomitant PI3K-AKT and p53 alterations in endometrial carcinomas are associated with poor prognosis. Mod Pathol 22: 522-529, 2009.

23. Konopka B, Janiec-Jankowska A, Kwiatkowska E, Najmoła U, Bidziński M, Olszewski W and Goluda C: PIK3CA mutations and amplification in endometrioid endometrial carcinomas: Relation to other genetic defects and clinicopathologic status of the tumors. Hum Pathol 42: 1710-1719, 2011.

24. German S, Aslam HM, Saleem S, Raees A, Anum T, Alvi AA and Haseeb A: Carcinogenesis of PIK3CA. Hered Cancer Clin Pract 11: 5, 2013

25. English DP, Bellone S, Cocco E, Bortolomai I, Pecorelli S, Lopez S, Silasi DA, Schwartz PE, Rutherford T and Santin AD: Oncogenic PIK3CA gene mutations and HER2/neu gene amplifications determine the sensitivity of uterine serous carcinoma cell lines to GDC-0980, a selective inhibitor of Class I PI3 kinase and mTOR kinase (TORC1/2). Am J Obstet Gynecol 209: 465. e1-e9, 2013.

26. Dong Y, Yang X, Wong O, Zhang X, Liang Y, Zhang Y, Wong W, Nong L, Liao Q and Li T: PIK3CA mutations in endometrial carcinomas in Chinese women: Phosphatidylinositol 3'-kinase pathway alterations might $t$ be associated with favorable prognosis. Hum Pathol 43: 1197-1205, 2012.

27. Herrero-Gonzalez S and Di Cristofano A: New routes to old places: PIK3R 1 and PIK3R2 join PIK3CA and PTEN as endometrial cancer genes. Cancer Discov 1: 106-107, 2011.

28. Lin DI: Improved survival associated with somatic PIK3CA mutations in copy-number low endometrioid endometrial adenocarcinoma. Oncol Lett 10: 2743-2748, 2015.

29. Wik E, Ræder MB, Krakstad C, Trovik J, Birkeland E, Hoivik EA, Mjos S, Werner HM, Mannelqvist M, Stefansson IM, et al: Lack of estrogen receptor- $\alpha$ is associated with epithelial-mesenchymal transition and PI3K alterations in endometrial carcinoma. Clin Cancer Res 19: 1094-1105, 2013.

30. Smith IN and Briggs JM: Structural mutation analysis of PTEN and its genotype-phenotype correlations in endometriosis and cancer. Proteins 84: 1625-1643, 2016.

31. Akiyama-Abe A, Minaguchi T, Nakamura Y, Michikami H, Shikama A, Nakao S, Sakurai M, Ochi H, Onuki M, Matsumoto K, et al: Loss of PTEN expression is an independent predictor of favourable survival in endometrial carcinomas. Br J Cancer 109: 1703-1710, 2013.

32. Djordjevic B, Hennessy BT, Li J, Barkoh BA, Luthra R, Mills GB and Broaddus RR: Clinical assessment of PTEN loss in endometrial carcinoma: Immunohistochemistry outperforms gene sequencing. Mod Pathol 25: 699-708, 2012. 
33. Catasus L, Gallardo A, Cuatrecasas $\mathrm{M}$ and Prat $\mathrm{J}$ : PIK3CA mutations in the kinase domain (exon 20) of uterine endometrial adenocarcinomas are associated with adverse prognostic parameters. Mod Pathol 21: 131-139, 2008.

34. Li W, Wang Y, Fang X, Zhou M, Li Y, Dong Y and Wang R Differential expression and clinical significance of DNA methyltransferase 3B (DNMT3B), phosphatase and tensin homolog (PTEN) and human MutL homologs 1 (hMLH1) in endometrial carcinomas. Med Sci Monit 23: 938-947, 2017.

35. Bradford LS, Rauh-Hain A, Clark RM, Groeneweg JW, Zhang L, Borger D, Zukerberg LR Growdon WB, Foster R and Rueda BR Assessing the efficacy of targeting the phosphatidylinositol 3kinase/AKT/mTOR signaling pathway in endometrial cancer. Gynecol Oncol 133: 346-352, 2014.

36. Morice P, Leary A, Creutzberg C, Abu-Rustum N and Darai E: Endometrial cancer. Lancet 387: 1094-1108, 2016.

37. Matias-Guiu X and Prat J: Molecular pathology of endometrial carcinoma. Histopathology 62: 111-123, 2013.

38. McIntyre JB, Nelson GS, Ghatage P, Morris D, Duggan MA, Lee CH, Doll CM and Köbel M: PIK3CA missense mutation is associated with unfavorable outcome in grade 3 endometrioid carcinoma but not in serous endometrial carcinoma. Gynecolc Oncol 132: 188-193, 2014.

39. Miyake T, Yoshino K, Enomoto T, Takata T, Ugaki H, Kim A, Fujiwara K, Miyatake T, Fujita M and Kimura T: PIK3CA gene mutations and amplifications in uterine cancers, identified by methods that avoid confounding by PIK3CA pseudogene sequences. Cancer Lett 261: 120-126, 2008

40. Garcia-Dios DA, Lambrechts D, Coenegrachts L, Vandenput I, Capoen A, Webb PM, Ferguson K; ANECS, Akslen LA, Claes B, et al: High-throughput interrogation of PIK3CA, PTEN KRAS, FBXW7 and TP53 mutations in primary endometrial carcinoma. Gynecol Oncol 128: 327-334, 2013

41. Tsikouras P, Bouchlariotou S, Vrachnis N, Dafopoulos A, Galazios G, Csorba R and von Tempelhoff GF: Endometrial cancer: Molecular and therapeutic aspects. Eu J Obstet Gynecol Reprod Biol 169: 1-9, 2013.

42. Janku F, Wheler JJ, Westin SN, Moulder SL, Naing A, Tsimberidou AM, Fu S, Falchook GS, Hong DS, Garrido-Laguna I, et al: $\mathrm{PI} 3 \mathrm{~K} / \mathrm{AKT} / \mathrm{mTOR}$ inhibitors in patients with breast and gynecologic malignancies harboring PIK3CA mutations. J Clin Oncol 30: 777-782, 2012

43. Bian X, Gao J, Luo F, Rui C, Zheng T, Wang D, Wang Y, Roberts TM, Liu P, Zhao JJ, et al: PTEN deficiency sensitizes endometrioid endometrial cancer to compound PARP-PI3K inhibition but not PARP inhibition as monotherapy. Oncogene 37: 341-351, 2018.

44. Philip CA, Laskov I, Beauchamp MC, Marques M, Amin O, Bitharas J, Kessous R, Kogan L, Baloch T, Gotlieb WH, et al: Inhibition of PI3K-AKT-mTOR pathway sensitizes endometrial cancer cell lines to PARP inhibitors. BMC Cancer 17: 638, 2017.

45. Hasegawa K, Kagabu M, Mizuno M, Oda K, Aoki D, Mabuchi S, Kamiura S, Yamaguchi S, Aoki Y, Saito T, et al: Phase II basket trial of perifosine monotherapy for recurrent gynecologic cancer with or without PIK3CA mutations. Invest New Drugs 35: 800-812, 2017.

46. Mirantes C, Dosil MA, Eritja N, Felip I, Gatius S, Santacana M, Matias-Guiu X and Dolcet X: Effects of the multikinase inhibitors Sorafenib and Regorafenib in PTEN deficient neoplasias. Eur J Cancer 63: 74-87, 2016.

47. McCormick A, Earp E, Leeson C, Dixon M, O'Donnell R, Kaufmann A and Edmondson RJ: Phosphatase and Tensin Homolog is a potential target for ovarian cancer sensitization to cytotoxic agents. Int J Gynecol Cancer 26: 632-639, 2016.

48. Tsiatis AC, Norris-Kirby A, Rich RG, Hafez MJ, Gocke CD, Eshleman JR and Murphy KM: Comparison of Sanger sequencing, pyrosequencing, and melting curve analysis for the detection of KRAS mutations: Diagnostic and clinical implications. J Mol Diagn 12: 425-432, 2010
49. Ihle MA, Fassunke J, König K, Grünewald I, Schlaak M, Kreuzberg N, Tietze L, Schildhaus HU, Büttner R and Merkelbach-Bruse S: Comparison of high resolution melting analysis, pyrosequencing, next generation sequencing and immunohistochemistry to conventional Sanger sequencing for the detection of p.V600E and non-p V600E BRAF mutations. BMC Cancer 14: 13, 2014

50. Li BS, Wang XY, Ma FL, Jiang B, Song XX and Xu AG: Is high resolution melting analysis (HRMA) accurate for detection of human disease-associated mutations? A meta analysis. PLoS One 6: e28078, 2011.

51. Vossen RH, Aten E, Roos A and den Dunnen JT: High-resolution melting analysis (HRMA): More than just sequence variant screening. Hum Mutat 30: 860-866, 2009.

52. Colombo N, Creutzberg C, Amant F, Bosse T, González-Martín A, Ledermann J, Marth C, Nout R, Querleu D, Mirza MR, et al: ESMO-ESGO-ESTRO consensus conference on endometrial cancer diagnosis, treatment and follow-up. Int J Gynecol Cancer 26: 2-30, 2016.

53. Kafshooz L, Kafshdooz T, Tabrizi AD, Mohaddes Ardabili SM, Ardabili M, Arkbazadeh A, Gharesouran J, Ghojazadeh M and Farajnia S: Role of exon 7 PTEN gene in endometrial carcinoma. Asian Pac J cancer Prev 16: 4521-4524, 2015.

54. Simi L, Pratesi N, Vignoli M, Sestini R, Cianchi F, Valanzano R, Nobili S, Mini E, Pazzagli M and Orlando C: High-resolution melting analysis for rapid detection of $K R A S, B R A F$, and PIK $3 C A$ gene mutations in colorectal cancer. Am J Clin Pathol 130: 247-253, 2008

55. Ousati AshtianiZ,Mehrsai AR,Pourmand MR and Pourmand GR: High resolution melting analysis for rapid detection of PIK3CA gene mutations in bladder cancer: A mutated target for cancer therapy. Urol J 15: 26-31, 2018.

56. Martínez-Carretero C, Pascual FI, Rus A and Bernardo I: Detection of EGFR mutations in patients with non-small cell lung cancer by high resolution melting. Comparison with other methods. Clin Chem Lab Med 55: 1970-1978, 2017.

57. Tsiatis AC, Norris-Kirby A, Rich G, Hafez MJ, Gocke CG, Eshleman JR and Murphy KM: Comparison of Sanger sequencing, pyrosequencing, and melting curve analysis for the detection of KRAS mutations: Diagnostic and clinical implications. J Mol Diagn 12: 425-432, 2010

58. Engelman A: Targeting PI3K signaling in cancer: Opportunities, challenges and limitations. Nat Rev Cancer 8: 550-562, 2009

59. Di Nicolantonio F, Arena S, Tabernero J, Grosso S, Molinari F, Macarulla T, Russo M, Cancelliere C, Zecchin D, Mazzucchelli L, et al: Deregulation of the PI3K and KRAS signaling pathways in human cancer cells determines their response to everolimus. J Clin Invest 120: 2558-2866, 2010.

60. Ni X, Zhang W and Huang RS: Pharmacogenomics discovery and implementation in genome-wide association studies era. Wiley Interdiscip Rev Syst Biol Med 5: 1-9, 2013.

61. Tsimberidou AM, Iskander NG, Hong DS, Wheler JJ, Falchook GS, Fu S, Piha-Paul S, Naing A, Janku F, Luthra R, et al: Personalized medicine in a phase I clinical trials program: The MD Anderson Cancer Center initiative. Clin Cancer Res 15: 6373-6383, 2012.

62. Westin SN, Ju Z, Broaddus RR, Krakstad C, Li J, Pal N, Lu KH, Coleman RL, Hennessy BT, Klempner SJ, et al: PTEN loss is a context-dependent outcome determinant in obese and non-obese endometrioid endometrial cancer patients. Mol Oncol 9: $1694-1703,2015$

This work is licensed under a Creative Commons Attribution-NonCommercial-NoDerivatives 4.0 International (CC BY-NC-ND 4.0) License. 